\title{
Motivación del estudiante: la diferencia entre parecer y ser buen estudiante
}

Fecha de recepción: 25 de noviembre de 2016 Fecha de aprobación: 22 de diciembre de 2016 Pp. 45-80
Lina María García Méndez ${ }^{l}$

Universidad EAN

lgarciam9990@universidadean.edu.co

María Alejandra Rolón Buenhaber ${ }^{2}$

Universidad EAN

mrolonbu8192@universidadean.edu.co

Cómo citar este artículo: García, L. M. y Rolón, M. A. (2016). Motivación del estudiante: la diferencia entre parecer y ser buen estudiante. Comunicación, cultura y política, 7, pp. 45-80.

\section{Resumen}

Aprender a escribir y leer correctamente, como adquirir cualquier otra habilidad o conocimiento, necesita práctica y disciplina suficientes que demostrarán en últimas que todo proceso de aprendizaje exige un trabajo autónomo constante. Si bien el apoyo en estos procesos en el ámbito académico es fundamental, el conocimiento se consolida de forma personal y para ello es necesario el trabajo independiente. En este artículo, se pretende indagar sobre las razones por las cuales los estudiantes asisten al Centro de Lectura y Escritura Académica de la Universidad EAN.

Aunque la mayoría de los testimonios muestran que los interesados, que por lo general tienen debilidad a la hora de escribir, acuden para mejorar por medio de la tutoría de pares; sin embargo, se cree que pueden existir otra serie de motivos por los cuales un estudiante decide tomar las tutorías.

\section{Palabras clave}

Lectura, escritura, estudiantes, tutorías

\footnotetext{
${ }^{1}$ Estudiante de Lenguas Modernas en la Universidad EAN. Técnico en Seguridad Ocupacional - Servicio Nacional de Aprendizaje SENA.

${ }^{2}$ Estudiante de Lenguas Modernas en la Universidad EAN.
} 


\title{
Student motivation: the difference between appearing to be a good student and actually being a good student
}

\begin{abstract}
Learning to write and read correctly, such as acquiring any other skill or knowledge, requires enough practice and discipline that will ultimately demonstrate that every learning process requires constant autonomous work. Although the support in these processes in the academic field is fundamental, knowledge is consolidated in a personal way and, to that end, independent work is necessary. This article aims to inquire about the reasons why students attend the Academic Reading and Writing Center of the EAN University.

Although most of the testimonies show that those who are interested, students who usually have some sort of weakness when writing, come to improve through peer tutoring; however, it is believed that there may be another set of reasons why a student decides to take tutoring.
\end{abstract}

\section{Keywords}

Reading, writing, students, tutoring.

\section{Motivation estudiantine: la différence entre l'esprit et être un bon élève}

\section{Résumé}

Apprendre à écrire et à lire correctement, acquérir de nouvelles aptitudes et connaissances requiert d'une pratique et d'une discipline exigeantes qui révèlent que tout processus d'apprentissage exige un travail personnel constant. Si le soutien scolaire lors de ce processus académique est fondamental, la connaissance se consolide de manière personnelle et le travail autonome devient primordial. Cet article prétend montrer les raisons pour lesquelles les étudiants assistent au Centre de lecture et d'écriture académique de l'Université EAN. Si la majorité des témoignages rendent compte que les étudiants ont des lacunes en production écrite qu'ils comblent grâce à des séances de tutorat, il semble exister d'autres motifs pour lesquels un étudiant décide de suivre ces séances de tutorats.

\section{Mots clés}

Lecture, écriture, étudiants, tutorats. 


\section{A motivação dos alunos: a diferença entre parecer e ser um bom aluno}

\section{Resumo}

Aprender a ler e escrever corretamente, assim como a aquisição de qualquer outra habilidade ou conhecimento, necessita prática e disciplina, o que demonstrará em última análise, que qualquer processo de aprendizagem exige trabalho autônomo constante. Embora o apoio a esses processos no âmbito acadêmico seja essencial, o conhecimento é consolidado de forma pessoal e isso exige um trabalho independente. Este artigo tem como objetivo investigar as razões pelas quais os alunos frequentam o Centro de leitura e escrita acadêmica da universidade EAN. Embora a maioria dos depoimentos mostram que os interessados, que normalmente têm deficiência na escrita, recorrem para melhorar através de tutoria entre pares, no entanto, acredita-se que pode haver um outro conjunto de razões pelas quais um estudante decide tomar as tutorias.

\section{Palavras-chave}

Leitura, escrita, estudantes, tutorias. 


\section{Introducción}

T n el ámbito universitario, existe la problemática de que los estudiantes ingresan con un grado de preparación inferior al que se espera (Ordóñez, 2017; Cuervo, 2013). Sin mencionar que las competencias, específicamente de lectura, escritura y redacción, no están desarrolladas plenamente. Por ello, surgen los centros de escritura, cuyo objetivo es desarrollar y fortalecer las habilidades de escritura y lectura de los estudiantes universitarios, por medio de la tutoría entre pares.

A inicios de 2016, en la Universidad EAN se formó el Centro de Lectura y Escritura Académica, cuyo propósito es guiar a los estudiantes en el proceso de escritura. Los estudiantes llegan a la universidad con vacíos en cuanto al cómo escribir un texto cohesivo y coherente, y por ello se generan espacios en los que el estudiante pueda resolver sus dudas y, además, mejorar siendo consciente de sus principales errores.

En estos espacios, es fundamental la intención de aprender cosas nuevas por voluntad propia. Y ya que los estudiantes llegan con falencias, lo más sensato sería pensar que la mayoría de ellos necesitan estas tutorías. Sin embargo, hay que tener en cuenta dos aspectos importantes de aquellas: el primero es que son relativamente pocas, en parte porque este espacio es nuevo; y segundo, porque un porcentaje los estudiantes no están interesados en asistir, incluso si son conscientes de sus vacíos.

Otro aspecto es que se entiende el estudio autónomo como uno en el que la figura del profesor aún es fundamental, y de ella depende en buena medida el proceso de aprendizaje. Encontramos que el estudio autónomo es fundamental para el proceso de adquisición de conocimientos. No obstante, en esta investigación, fueron predominantes los escritos elaborados por maestros, quienes identifican con facilidad las falencias de los estudiantes. Los que consultamos fueron:

- Soledad Aguilera M. y Yanina Elizabet Boatto con su artículo "Seguir escribiendo... seguir aprendiendo: la escritura de textos académicos en el nivel universitario", quienes recuperan una serie de aportes teóricos sobre la 
producción de textos académicos en sugerencia al desarrollo de la escritura en los estudiantes.

- Dyanne Escorcia, Mayilín Moreno, Kiara Campo y Jorge Palacio con su artículo "Enseñanza y evaluación de la escritura en la universidad: análisis de prácticas declaradas de docentes franceses y colombianos", quienes analizan las representaciones de los docentes universitarios sobre sus prácticas de enseñanza y de evaluación de la escritura académica.

- Alfonso Rodríguez M., Elcira Solano, Armando Martínez y Luisa del Villar H. con su artículo "Lectura, escritura y calidad en la universidad colombiana", quienes explican los propósitos con los que se escribe y se lee en la universidad, y cómo tiene implicaciones en la calidad educativa.

Estos docentes, al no estar al mismo nivel que el de los aprendices, pueden ver con una perspectiva diferente de la que tienen estos los factores que influyen en las actitudes que tales investigadores encuentran. Como estudiantes, al estar en la misma posición de las personas que estudiamos, pretendemos, desde una visión equivalente a la de ellos, encontrar aquellas razones que los impulsan a acudir a este centro.

Entonces, desde el semillero de investigación Retórica, argumentación y discurso, decidimos hacer esta investigación aplicada a este centro. Para ello, contamos solo con un mes y medio, por lo cual decidimos enfocarnos primordialmente en la perspectiva de los estudiantes asistentes y también de algunos que no han asistido.

En este artículo, presentamos inicialmente la recopilación teórica necesaria para el desarrollo de la investigación y su respectiva comprensión. A partir de allí, explicamos la metodología que utilizamos a fin de desarrollar esta investigación, que incluye su objetivo, la elección de participantes y los métodos de recolección de información. Posteriormente, presentamos los resultados y el respectivo análisis de lo que encontramos para dar paso, finalmente, a las conclusiones. 


\section{Marco teórico}

$7 \mathrm{n}$ esta investigación, partimos del hecho de que la mayoría de las universidades promueven la lectura y la escritura. Los centros de escritura se crean primeramente en los Estados Unidos alrededor de 1970, donde se buscaba la adquisición de habilidades de escritura y lectura en las personas interesadas en ingresar a la universidad, pero que no las tenían. Hacia la década de 1990, se transformaron y su objetivo principal era hacer de ellos un lugar "donde todos los escritores en la comunidad universitaria pudieran convertirse, de manera reflexiva, en lectores competentes de su propia escritura"3 (Waller, 2002).

En Rhode Island College, en los Estados Unidos, se estudiaron en 2013 los procesos que se llevan a cabo en un programa, para entonces muy reciente y ampliamente usado por las universidades en ese país, denominado Directed Self-Placement, que funciona de manera similar a los centros de escritura actuales en Colombia. La diferencia es que este programa está habilitado solo para estudiantes de primer año, usualmente es obligatorio y los exámenes para evaluar la escritura son fundamentales. En el estudio, Caouette y Griggs (2015) explican que los estudiantes ingresan a la universidad con poca preparación, al menos en cuestiones lectoescritoras. "Many of RIC's first-year students are under-prepared for college writing (about half of the incoming class score 430 or below on the written and/or verbal portions of their SATs)"4, lo cual impulsa el uso de este tipo de programas de escritura en las universidades.

Aunque los centros de escritura actuales buscan una comunidad universitaria que sea competente en el ámbito de escritura y lectura, es innegable que aún existe la problemática de fondo de que los estudiantes ingresan poco preparados. Por tanto, es un reto para las universidades crear esa comunidad universitaria integral, donde la escritura y la lectura son fundamentales para el desarrollo profesional y personal.

\footnotetext{
3 Las traducciones son nuestras.

4 Muchos de los estudiantes de primer año de RIC están poco preparados para la escritura universitaria (aproximadamente la mitad de los entrantes obtuvieron en el examen SAT un puntaje de 430 o menor en las partes escritas y/o verbales).
} 
En los Estados Unidos, algunas universidades que manejan el programa de Directed Self-Placement son: California State University, University of Washington, Colorado State University, Wake Forest University, etc. En el caso de Colombia, la Pontificia Universidad Javeriana, la Universidad del Cauca, la Universidad de los Andes, la Universidad del Norte, la Universidad del Rosario, la Universidad Icesi, entre otras, cuentan con un centro de escritura que, cabe mencionar, es voluntario y funciona con la tutoría entre pares.

La Universidad EAN, por su parte, abre el Centro de Escritura y Lectura Académica en 2016. Su objetivo es potenciar la competencia lectora y escritora de los estudiantes. Lo más relevante es la tutoría de pares, la cual

permite que los escritores-alumnos aprendan a tomar las sugerencias de los lectores no como algo que debe cumplimentarse por acatamiento a una autoridad que califica, sino como comentarios a examinar y evaluar, que los ayuden a asumir su autoría, a decidir un propio plan de mejora del texto y, de este modo, a poder coordinar sus intenciones como autores con los efectos de sus textos sobre el lector. (Carlino, 2008).

La tutoría entre pares permite a los estudiantes expresarse con más libertad, y en esa medida les facilita comentar cuáles son sus dificultades y dudas frente a un tema específico. Asistir al centro es un acto totalmente autónomo por parte de los estudiantes, lo cual nos lleva a preguntarnos qué es la autonomía, a cual, según el Diccionario de la Real Academia Española, es la condición de quien, para ciertas cosas, no depende de nadie. De acuerdo con este concepto, profundizamos en lo que significa el aprendizaje autónomo en el entorno universitario, y según la Unesco (1999), citado por Peláez (2009, p. 4), el estudiante que aprende autónomamente es "un aprendiz que reconoce en las Tecnologías de Información y Comunicación, las bibliotecas y los ambientes universitarios en general, medios propicios para la construcción de conocimiento y deja el contacto docente".Y aquí es importante clarificar que dejar el contacto docente es pasar a poner a este actor como la persona encargada de potenciar el conocimiento, en lugar de mantenerlo como la fuente única de aprendizaje.

En la época actual, nueva sociedad de la información como la define Castells (1997), citado por Peláez (2009, p. 3), es más sencillo acceder al conocimiento de casi cualquier tema en el que se esté interesado, gracias a 
los avances tecnológicos. El joven moderno tiene mayor ventaja en el ámbito tecnológico sobre cualquier otro de la historia de la humanidad: la facilidad de adquirir cualquier recurso capaz de intercomunicar al mundo es manifestar y romper las fronteras y barreras en el ámbito de comunicación, financiero, diplomático, social y geográfico.

Por lo tanto, como dice Peláez (2009) "estudio autónomo (...) se entiende como ese esfuerzo mental o cognitivo que hace un sujeto con el fin de aprender algo", es decir, la labor realizada para la construcción de aprendizaje autónomo, entonces, el aprendizaje autónomo será el producto o efecto de dicho estudio.

Así, teniendo en cuenta todo lo mencionado, no solo es posible sino necesario que las personas estudien y aprendan autónomamente en esta época de fácil acceso a la información. "Se alcanza la autonomía cuando la persona llega a ser capaz de pensar por sí misma con sentido crítico, [...] se es autónomo cuando la persona es capaz de gobernarse a sí misma y es menos gobernado por lo demás" (Kamil, s. f., citado por Cárdenas, 2009, p. 8-9). Valdría la pena resaltar la diferencia entre aprendizaje autónomo y trabajo independiente. Este último "es cuando el alumno puede relacionar correctamente el planteamiento de la tarea con los medios a seguir para realizarla" (Yesipov, 1981, citado por Román y Herrera, 2010, p. 95). En este orden de ideas, el trabajo independiente sigue siendo aquel que es guiado por la figura del profesor, mientras que el autónomo son los esfuerzos que el estudiante hace por aprender sin completa necesidad de un docente.

En Colombia, se contempla el aprendizaje autónomo en el Decreto 1295, de 20 de abril de 2010 aún vigente. En la Universidad EAN, se puede evidenciar en el syllabus la cantidad de horas de trabajo autónomo que debe invertir el estudiante; en total 240 , de las cuales 150 son de trabajo autónomo. Sin embargo, cabe destacar:

Las instituciones educativas propenden en la actualidad a alcanzar un trabajo autónomo por parte de los estudiantes logrando aprendizajes significativos, pero lo que se evidencia en la cotidianidad y dentro de la práctica educativa es que los estudiantes aún presentan dificultades para pasar de un modelo de cátedra donde el dominio lo tiene el docente a un modelo autónomo con mayor compromiso por parte del estudiante. (Olaya y Quimbayo, 2014, p. 2) 
Los estudiantes son incapaces de poner sus ideas por escrito, "las causas de estas deficiencias suelen trasladarse a niveles previos del sistema educativo" (Vázquez, 2005, p. 5). Basados en que son los profesores de educación primaria y secundaria los que deben enseñar estrategias de escritura y lectura adecuadas, podría decirse que el proceso universitario se retrasa porque la universidad se está encargando de llenar los vacíos que dejó esta educación previa.

Cabe aclarar que la diferencia entre el centro de escritura que comenzó en los Estados Unidos y el actual es la perspectiva del estudiante frente al mundo más que el centro de escritura en sí. Esta perspectiva es una que tiene como base la era digital, ya mencionada, donde el estudiante tiene acceso a una innumerable cantidad de información y no siente la necesidad de buscar otro tipo de ayuda. Aun cuando sabe que tiene problemas, no ve la necesidad de asistir al centro, porque considera que con la información contenida en internet es suficiente.

Costará varios años llegar a una cultura del aprendizaje por motivación propia del estudiante, que consiste, de cierto modo, en que las personas aprendan a aprender. No se trata de llegar a una clase simplemente para volver a las mismas líneas de la lección convencional, donde el profesor es quien la dirige en todo momento, sino es más hacerles ver a los estudiantes que aprender es el medio de mantenerse adaptados en un mundo cambiante, en el cual es necesario tener herramientas que permitan reconocer las oportunidades que este nos brinda; y aquí podemos conectar la escritura con el pensamiento crítico. Al respecto, Vargas explica:

En el análisis de la relación escritura y pensamiento crítico, compartimos con Giroux (1997) que la escritura posee una doble vertiente de proceso interdisciplinario y de epistemología capaz de enseñar a los estudiantes a pensar crítica y racionalmente sobre una determinada materia. [...] Específicamente la pobreza en la escritura refleja, como plantea Giroux, pobreza de pensamiento, lo que lleva a concluir que los errores que presentan los estudiantes en sus composiciones escritas son de hecho errores en el proceso de elaboración del pensamiento. (2007, p. 25). 
Saber pensar críticamente no solo abre la posibilidad de ampliar el pensamiento individual, sino que crea sociedad, como bien explica Nussbaum (2015):

El pensamiento crítico es particularmente crucial para la buena ciudadanía en una sociedad que tiene que luchar a brazo partido con la presencia de personas que difieren según la etnia, la casta, la religión y profundas divisiones políticas. Solo tendremos la oportunidad de un diálogo adecuado que atraviese fronteras si los ciudadanos jóvenes saben cómo participar en el diálogo y la deliberación en primer lugar.

Para crear esta cultura mundial que sea capaz de enfrentar los problemas globales, son necesarias "la capacidad de pensar de manera crítica; la capacidad de trascender las lealtades locales y acercarse a los problemas mundiales como un 'ciudadano del mundo'; y la capacidad de imaginar comprensivamente la situación del otro" (Nussbaum, 2015). De esta manera, se crea humanidad, ya que nos enseñan a pensar por nosotros mismos y expandir nuestros horizontes mentales en un mundo donde las fronteras que nos hemos puesto durante la historia se están cayendo gradualmente.

Por otro lado, un concepto que vale la pena mencionar es el de marcador o patrón discursivo. Martín Zorraquino y Portolés (1999), citados por Mondaca, Méndez y Rivadeira (2015, p. 11), los define como "unidades lingüísticas invariables cuyo objetivo es el de guiar, de acuerdo con sus propiedades morfosintácticas, semánticas y pragmáticas, las inferencias que se realizan en la comunicación". Nuestro objetivo con estos marcadores es simplemente identificar si existen ciertos patrones de comportamiento que nos ayuden a corroborar nuestras suposiciones acerca de las razones por las cuales un estudiante busca ayuda en este centro.

\subsection{Objetivo de la investigación}

A partir de la pregunta "¿Cuáles son las verdaderas razones por las cuales un estudiante quiere acudir al Centro de Lectura y Escritura Académica?", pretendemos determinar si existen o no razones implícitas por las que pide ayuda y si estas tienen que ver con el aprendizaje autónomo; bien sea porque 
el estudiante hace uso de este último en sus formas de estudio, bien sea porque no lo hace y está impulsado a ir por motivos diferentes. Lo anterior, como ya mencionamos, en un límite de tiempo de mes y medio, y aplicado a este, que, hay que mencionar, solo hasta 2016 fue creado y no es conocido por todos.

\section{Metodología}

\subsection{Método e instrumento}

El método de recolección de información que utilizamos fue mixto, y empleamos la estrategia explicada por Creswell(2003) denominada Concurrent Nested Strategy, la cual combina la recolección de datos de tipo cuantitativo y cualitativo, en ella uno de los dos métodos es predominante y es el que guía el proyecto, mientras que el otro complementa el anterior; los datos obtenidos de ambos métodos son tenidos en cuenta en el análisis.

Neuman (1997, citado por Hossain, 2011, p. 147) expone las diferencias entre el método cuantitativo y cualitativo, y aunque se hizo uso de elementos de ambas partes, esta investigación es predominantemente cualitativa (Tabla 1).

Tabla 1. Diferencias entre el método cuantitativo y cualitativo

\begin{tabular}{|l|l|}
\hline \multicolumn{1}{|c|}{ Cuantitativo } & \multicolumn{1}{c|}{ Cualitativo } \\
\hline Medir hechos objetivos & Construir realidad social, significado cultural \\
\hline Centrado en variables & Enfoque en procesos y eventos interactivos \\
\hline La credibilidad es clave & La autenticidad es clave \\
\hline Libre de juicios de valor & Los juicios de valor están presentes y son explícitos \\
\hline Independiente del contexto & Limitado a las circunstancias y situaciones \\
\hline Varios casos, temas & Pocos casos, temas \\
\hline Análisis estadístico & Análisis temático \\
\hline Investigador imparcial & El investigador está involucrado \\
\hline
\end{tabular}

Fuente. Hossain (2011). 
Entonces, para la recolección de información, se decidió hacer uso de la entrevista y la encuesta. Con esta última, se buscó principalmente indagar sobre las preguntas base que dieran paso a otras en las que, en la entrevista, se pudieran profundizar. Se hizo de esta manera porque consideramos que la encuesta arroja datos generales en los que por su formato no se puede profundizar ya que no hay interacción. Por ende, desde los primeros resultados que obtuvimos, los de la encuesta, formulamos preguntas en las que pudiéramos obtener respuestas más específicas.

Además, con autorización de sus miembros, utilizamos los resultados de las encuestas que realiza este centro a todos los asistentes y a los tutores. Lo anterior para tener una idea general del tema de consulta recurrente en las tutorías y también los datos estadísticos que refieren la asistencia por facultad, carrera y materia.

Hay que manifestar que la investigación es esencialmente cualitativa, porque, como explicamos, el método que guía el proyecto es el cualitativo, por lo cual las entrevistas fueron fundamentales para nuestro análisis. Para ellas, utilizamos preguntas básicas que lograran que los estudiantes se sintieran más cómodos y respondieran con mayor libertad y sinceridad. La entrevista fue semiestructurada, pues, aunque teníamos unas preguntas base, también pudimos formular algunas de acuerdo con las respuestas que íbamos obteniendo, lo cual permitió conducirla más como una conversación sin presiones. Todas las respuestas tienen autorización de ser expuestas y utilizadas en este artículo protegiendo la confidencialidad.

Al ser una investigación predominantemente cualitativa, es importante resaltar que, como explica Creswell (2003), el método cualitativo es en lo fundamental interpretativo, es decir que el investigador hace una interpretación de los resultados. Aquí se incluye la descripción de un caso o una situación, el análisis por temas o categorías y, por último, hacer una interpretación o presentar las conclusiones sobre su significado personal y, teóricamente, mencionando lo que se aprendió y formulando posibles preguntas que podrían resolverse después. Así es como los juicios de valor personales son fundamentales en este tipo de investigaciones, al igual que es determinante el contexto social 
e histórico en el que se sitúan los autores. Al respecto, Creswell (2003): "It also means that the researcher filters the data through a personal lens that is situated in a specific sociopolitical and historical moment. One cannot escape the personal interpretation brought to qualitative data analysis"s.

Indagamos sobre los métodos de lectura y escritura que utilizan los estudiantes en su cotidianidad, con el fin de identificar si después de asistir a las tutorías continuaban trabajando sobre sus falencias o si simplemente querían asegurar una buena nota en un trabajo específico.

\subsection{Selección de participantes}

Se envió la encuesta solo a personas que ya habían asistido a este centro, en total diecisiete, y como resultado obtuvimos cinco respuestas. El periodo al que nos estamos refiriendo parte del 19 de agosto al 9 de noviembre de 2016 . Por su parte, la entrevista fue realizada a cuatro personas que acudieron al centro, que incluye a dos que habían solicitado sus servicios más de una vez (esta última es una situación poco común), a la tutora con más antigüedad del centro y a diez personas de distintas carreras de la universidad que no han asistido a este centro, con el fin de obtener sus opiniones (Tabla 2).

5 Esto también significa que el investigador filtra la información a través de una visión personal situada en un momento sociopolítico e histórico específico. En el análisis de datos cualitativos no se puede evadir la interpretación personal. 
Tabla 2. Participantes

\begin{tabular}{|c|c|c|c|c|}
\hline & Género & Carrera & Semestre & $\begin{array}{l}\mathrm{N}^{\circ} \text { de } \\
\text { asistencias a } \\
\text { este centro }\end{array}$ \\
\hline \multirow{2}{*}{ Tutores } & Femenino & Lenguas Modernas & 4 & - \\
\hline & Masculino & Lenguas Modernas & 6 & - \\
\hline \multirow{4}{*}{ Asistentes } & Femenino & Lenguas Modernas & 4 & 2 \\
\hline & Masculino & Lenguas Modernas & 3 & 1 \\
\hline & Femenino & Lenguas Modernas & 1 & 1 \\
\hline & Femenino & Ingeniería Ambiental & 4 & 2 \\
\hline \multirow{9}{*}{$\begin{array}{l}\text { No. } \\
\text { asistentes }\end{array}$} & Masculino & Administración de Empresas & 2 & - \\
\hline & Masculino & Ingeniería de Sistemas & 4 & - \\
\hline & Masculino & Ingeniería Ambiental & 4 & - \\
\hline & Masculino & Lenguas Modernas & 4 & - \\
\hline & Masculino & Lenguas Modernas & 4 & - \\
\hline & Masculino & Ingeniería de Energías & 3 & - \\
\hline & Masculino & Ingeniería de Energías & 2 & - \\
\hline & Femenino & Estudios y Gestión Cultural & 6 & - \\
\hline & Femenino & Negocios Internacionales & 3 & - \\
\hline
\end{tabular}

Fuente. Elaboración propia.

Se tabularon las respuestas de las encuestas para tener una visión más general de ellas. Además, examinamos los resultados de la encuesta del centro, que está orientada a saber la percepción del estudiante y del tutor con respecto a la sesión de tutoría, y tomamos la información de aquellas preguntas que eran fundamentales para el análisis de los resultados que obtuvimos. Estas son presentadas a lo largo de los resultados.

En cuanto a las entrevistas, el proceso que llevamos fue, inicialmente, buscar personas que hayan asistido al centro, a los tutores y a varias personas de diferentes carreras que no han solicitado colaboración de este. Para el desarrollo de la entrevista, iniciábamos explicándoles a los participantes el motivo por el cual pedíamos su colaboración, presentábamos un preámbulo de lo que es el semillero de investigación y especificábamos que sus respuestas iban a ser grabadas para luego preguntar si nos permitían utilizarlas, no sin antes manifestar que su identidad iba a permanecer en anonimato. A partir de allí, como mencionamos, la entrevista se daba de forma muy conversacional para 
que los participantes hablaran sin reservas, temores o cualquier impedimento que evitara que sus respuestas fueran lo más espontáneas posible. Una vez terminadas, transcribimos cada una de ellas, para analizar las respuestas, y aquellas que pudieran repetirse fueran agrupadas, y así encontrar patrones en el discurso de los estudiantes.

\section{Resultados}

Dresentamos inicialmente los resultados de las preguntas de sí y no de esta E encuesta (figura 3). En primer lugar, indagamos sobre si los estudiantes creen necesaria la asistencia constante a este centro con el fin de encontrar si sus intenciones eran solo la corrección de un trabajo específico o mejorar sus falencias en general, ya que esto requeriría una asistencia, como se ha dicho, constante (Figura 1).

Figura 1. Preguntas cerradas.

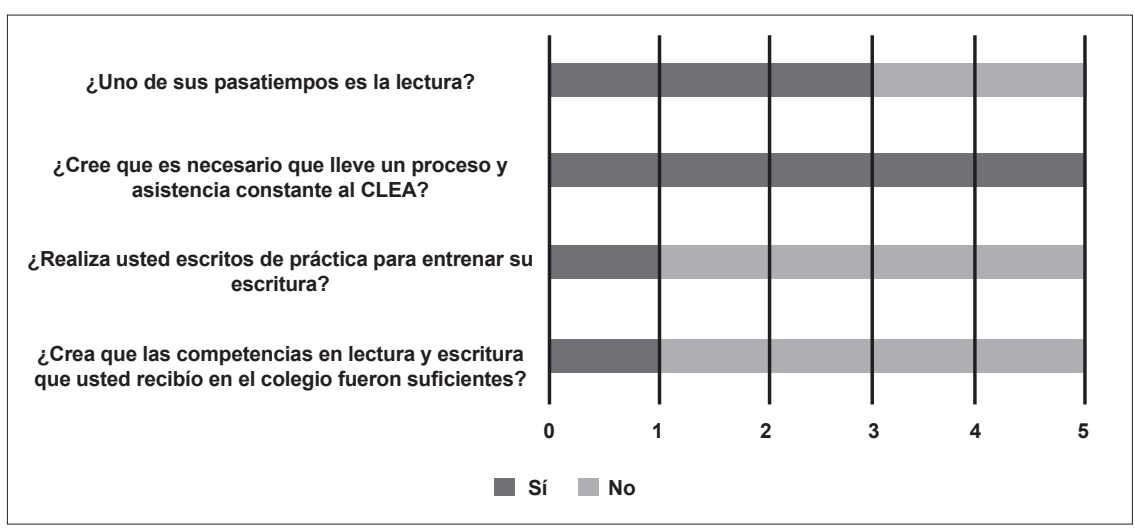

Fuente. Elaboración propia.

Todos los estudiantes encuestados confirmaron estar satisfechos con los resultados de las tutorías y también con los comentarios que les hicieron los tutores a cargo. Ellos, al evaluar a los asistentes, llegaron a la conclusión de que todos necesitaban un proceso de asistencia constante (Figura 2). 
Figura 2. ¿Se cumplieron sus expectativas con la tutoría?

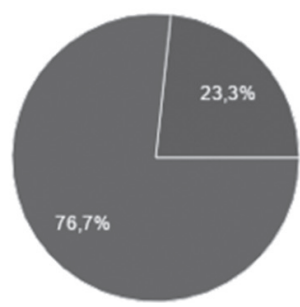

Totalmente de acuerdo

De acuerdo

En desacuerdo

Totalmente en desacuerdo

Fuente. Elaboración propia.

\subsection{Método de análisis de datos}

En primer lugar, paradójicamente, a pesar de que los estudiantes que respondieron a esta encuesta afirman necesitar más de una tutoría, solo $5 \%$ ha pedido una segunda tutoría (Figura 3).

Figura 3. Respuesta obtenida de las encuestas del Centro de Lectura y Escritura Académica

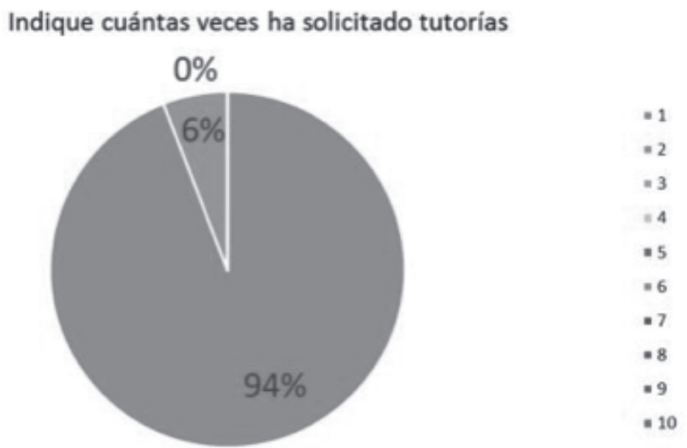

Fuente. Elaboración propia. 
En segundo lugar, se indagó sobre los hábitos de lectura de los estudiantes, con el fin de identificar si hay relación entre la lectura y las razones para ir a este centro, teniendo en cuenta el modelo de aprendizaje de escritura propuesto por Rojas-García (2016), que "busca orientar a los estudiantes en la producción de un texto a partir de las habilidades adquiridas en los procesos de lectura" (p. 197).

A pesar de que el contenido que se lee es muy variado (Figura 4), hay que tener en cuenta que los estudiantes afirmaron leer, sobre todo, noticias. Vega (2015, p. 3) señala que después de una encuesta realizada a más de 2000 personas entre los 12 y 29 años, "los jóvenes sí leen y lo hacen por gusto. No obstante, los contenidos que consumen son cada vez más breves y efímeros y no siempre abarcan lo que se define como literatura 'culta"' (Vega, 2015, p. 3). Además, hay que manifestar que estos contenidos son los que principalmente encuentran en línea. Al respecto, este autor indica: "Los chavos leen chats, blogs, tutoriales, cómics, y lo hacen en su convivencia en redes sociales o en Internet, sobre todo a través de sus teléfonos móviles" (Vega, 2015, p. 3).

Figura 4. ¿Cuáles de las siguientes opciones hacen parte del contenido que lee?

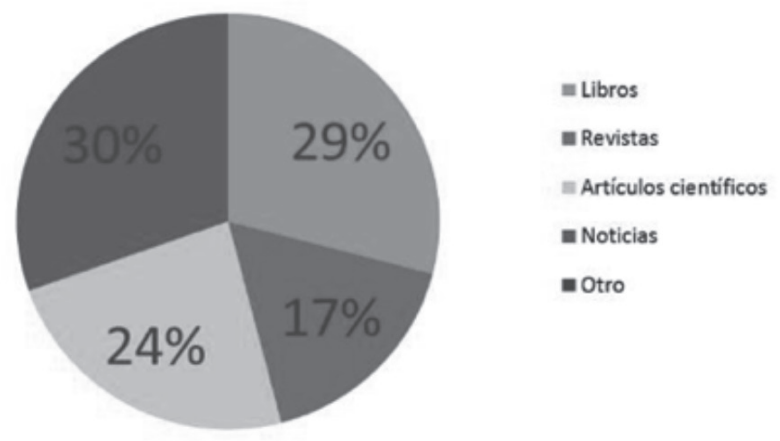

Fuente. Elaboración propia.

En tercer lugar, preguntamos si suelen escribir como entrenamiento para mejorar, con el objetivo de establecer si, gracias a su práctica, llegaban a la tutoría con dudas puntuales y si, debido a su interacción constante con la escritura, su presencia en el centro es por el deseo de aprender cosas nuevas. 
En cuarto lugar, la pregunta iba dirigida a saber si ellos consideran que la enseñanza que recibieron del colegio en cuanto a lengua materna fue buena o suficiente para su proceso de lectoescritura, con el objeto de identificar si consideraban tener vacíos respecto de la redacción y si estaban dispuestos a llenarlos.

De acuerdo con lo anterior, es en las siguientes áreas de estudio (Figura 5), en las que los estudiantes caen en la cuenta de que tienen falencias.

Figura 5. Unidad de estudio.

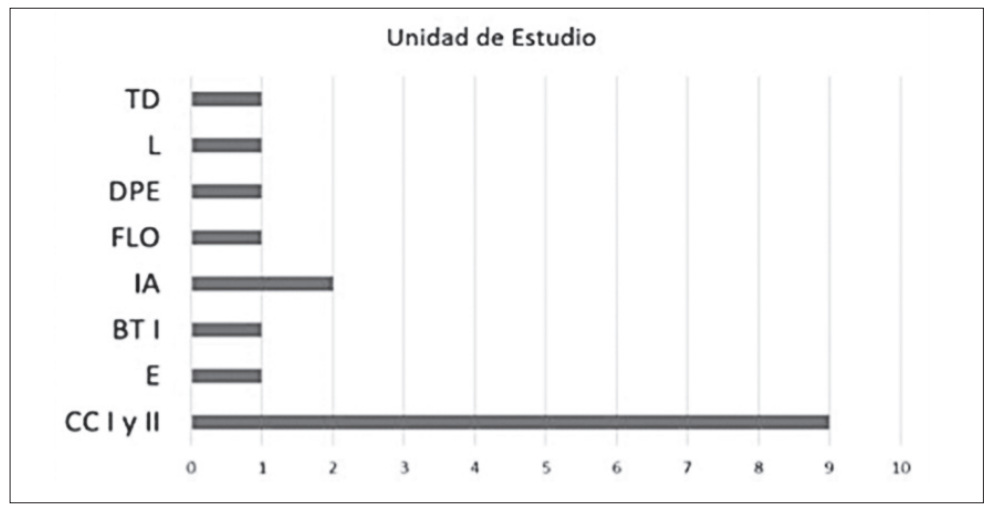

TD: teoría discursiva; L: lexicología; DPE: desarrollo personal para empresarios; FLO: fundamentos de lingüística organizacional; IA: introducción a la administración; BT I: business translation I; E: economía; CC I y II: competencias comunicativas I y II.

Fuente. Elaboración propia.

Es precisamente en competencias comunicativas I y II donde a los estudiantes se les pide redactar textos académicos, pues este es el fin de tales unidades de estudio. Cabe preguntarse si la exigencia de buena redacción en materias diferentes de estas últimas es más débil. Tal vez debido a que no en todas las materias se exige el mismo nivel de lectura y escritura, los estudiantes no ven la necesidad de mejorar este tipo de habilidades.

Dentro de las preguntas abiertas, estaba incluida “¿Por qué decidió acudir al Centro de Lectura y Escritura Académica?", para la cual la respuesta general obtenida expresaba las preocupaciones en cuanto a redacción, específicamente en ensayos. Con este mismo tipo de pregunta, indagamos también sobre 
cuánto tiempo manifiestan invertir para estudiar los temas de cada materia. Los resultados obtenidos mostraban que los estudiantes invertían de tres a cinco horas, y que, en general, debido a malos resultados en los escritos que presentaban en las clases, fueron conscientes del problema y, por eso, se motivaron a asistir a este centro. En este punto, es conveniente clarificar que el poco interés que algunos estudiantes muestran sobre este centro se ve reflejado en la falta de atención que prestaron a las encuestas o a atender a los correos que enviamos para contactarlos.

Por este motivo, fue necesario contactar a personas, que aunque no asistieron a este centro, podían darnos la percepción que tienen de él y también por qué no se han visto involucrados en la tarea de asistir. Para ello, consideramos reunir y entrevistar estudiantes de las diferentes carreras de la Universidad EAN, porque es importante analizar si los distintos estudios en los que se enfocan influyen en ciertos pensamientos que tienen, que ampliaremos más adelante.

Entonces, de manera general, un punto en común en el pensamiento de ambos grupos de entrevistados es que es mucho más sencillo y cómodo asistir a una tutoría con otro estudiante que con un profesor, porque hay mayor facilidad de comunicación entre ellos y la posibilidad de ser juzgados se reduce.

Otro rasgo compartido es que todos consideran importante la existencia de este centro, unos en mayor medida que otros, pero están de acuerdo en que para la comunidad universitaria es un espacio muy útil que si se sabe aprovechar puede dar buenos resultados. Se entiende que el centro está abierto a todos los estudiantes sin importar la carrera que cursan, porque cualquiera puede necesitarlo.

Una vez obtenidas las opiniones de los estudiantes asistentes, tomamos también la palabra de dos de los tres tutores del centro, pues conocer la percepción que ellos tienen, de acuerdo con el proceso de los estudiantes a quienes ayudan, nos permite confirmar si realmente lo que ocurre es como lo describe el aprendiz. 
Como se indicó, se tuvieron en cuenta los resultados de las encuestas que ha realizado este centro. Esta vez se citarán los reportes de los tutores con respecto a las tutorías que han hecho (Figura 6).

Figura 6. Compromisos estudiante-tutor.

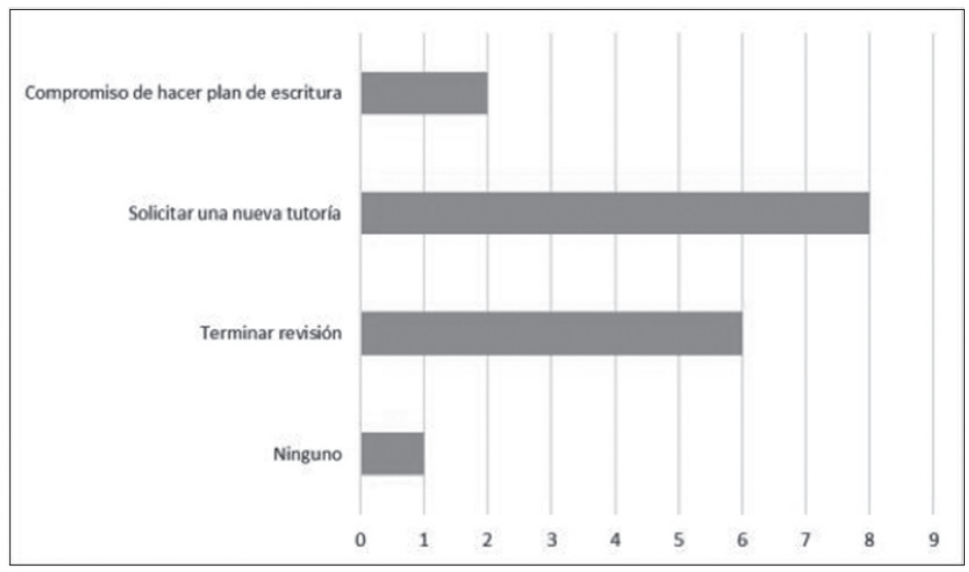

Fuente. Elaboración propia.

Siguiendo esta línea, podemos manifestar que en las entrevistas con los tutores encontramos que más de la mitad de los estudiantes necesitan más de una sesión para mejorar en el proceso de escritura y, sin embargo, ninguno vuelve a reservar una tutoría. También debemos tener en cuenta que durante la sesión los estudiantes se comprometen con los tutores a seguir un proceso con el centro; aun así, los tutores en la entrevista aseguraron que en su mayoría los aprendices acuden por obtener buenas calificaciones en los trabajos que van a presentar y, por ello, necesitan ayuda en un trabajo específico. Por tanto, las intenciones de mejorar las habilidades de redacción quedan en un segundo plano y prevalece el interés de obtener buenas calificaciones sin tomar en cuenta si se aprendió o no.

Uno de los factores determinantes de si los estudiantes van realmente a mejorar sus habilidades o simplemente por obtener una buena calificación es su actitud durante la tutoría. La tendencia de los que están realmente interesados es la de ser propositivos, estar muy dispuestos y pendientes de las observaciones de los tutores y la frecuencia con la que van a las tutorías. Por 
otro lado, los estudiantes con poco interés en mejorar sus habilidades tienden a llegar sin preguntas, a dispersarse con facilidad y a buscar excusas para que el tutor termine haciendo todo el trabajo.

También podemos considerar un punto medio. Uno de los estudiantes que se entrevistó y que asiste al centro presentaba ciertas incoherencias en su discurso. En la entrevista, hizo evidente su interés por asistir y mejorar, no obstante, su interés no iba más allá, porque afirmaba no tener tiempo suficiente ni para asistir ni para hacer ejercicios de práctica de escritura o lectura (posteriormente ampliaremos las incoherencias en los argumentos de falta de tiempo). Lo anterior genera que su proceso de mejora se vea retrasado, y, como lo expresaron los tutores en la entrevista, es necesario el ejercicio constante de la escritura para su mejoría. Al ser esta una habilidad, lo más factible es que mediante la práctica se desarrolle óptimamente.

Los estudiantes que van con poco interés al centro no lo expresan abiertamente. Uno de los entrevistados tuvo una tutoría a la cual no llevó ningún texto para analizar. Si tenemos en cuenta que uno de los requisitos que piden los tutores es llevar un texto de la autoría del interesado para poder colaborarle, podríamos manifestar que el estudiante en cuestión no tenía el interés suficiente y tampoco leyó atentamente los correos.

Asimismo, pudimos comprobar que varios de los estudiantes prefieren guardar las apariencias con respecto a los conocimientos que poseen. Un ejemplo es que uno de ellos afirmó aprender palabras que tienen un registro alto para que en el momento de usarlas pareciera una persona mucho más culta aun cuando sabe que no lo es. Aquí su testimonio: "Digamos, yo cojo palabras de las que... no sé, como por ejemplo verborrea, cuando quiero utilizarlas en contra de alguien, para que la gente diga 'iay este tipo sabe harto!'”.

Otro estudiante que señala leer una cantidad considerablemente alta de libros, artículos, revistas, periódicos, etc., dice saber que tiene problemas de redacción, pero no sabe identificar sus propias falencias. También indica que escribe por gusto, de forma recurrente, lo cual hace que su discurso sea contradictorio, ya que, como se dijo antes, sin práctica no hay avances. Si bien el estudiante gusta de escribir y lee bastante, aún no es consciente de la importancia de la revisión de un tercero que pueda brindar una nueva 
perspectiva a sus escritos académicos. Pues, como nos dejaron ver los tutores, la mejor manera de darnos cuenta de un error es cuando se pide la revisión de otra persona.

Por lo que se refiere a la asistencia de los estudiantes a este centro, siempre encuentran una excusa para justificar su inasistencia. La que más predomina es la falta de tiempo. Un estudiante que asistió al centro afirma no seguir proceso porque su horario es ajustado. En contraste, la Universidad EAN plantea los syllabus de las unidades de estudio con una intensidad horaria muy específica. Por un lado, las horas de acompañamiento directo, y por el otro, las horas de trabajo independiente. Por ejemplo, una unidad de cinco créditos académicos tiene 240 horas: 90 de acompañamiento directo y 150 de trabajo independiente, es decir, los estudiantes deberían dedicar una hora diaria de sus rutinas a trabajo independiente.

No obstante, esta investigación no solo demuestra que no lo hacen, porque para ellos trabajo independiente solo tiene lugar cuando se ve en riesgo la nota de una unidad de estudio, cuando no se entiende nada del contenido presentado en ella, por ejemplo: "Yo estudiaba para matemáticas, que era la materia en la que más mal me iba, pero cuando me di cuenta de que no entendía nada, dejé de hacerlo. Y ahora sé que ya me tiro la materia"; o cuando va a tener lugar un parcial, uno de ellos indica: "Estudio si tengo parcial, si no, no". Y aquí otro testimonio: "Poco estudio temas relacionados con la universidad. En este momento, no estoy estudiando. En general, hago las tareas, pero no dedico tiempo a estudiar propiamente".

Cabe anotar que indagamos sobre qué cosas hacen en su tiempo libre y muchos lo emplean para dormir, para estar en redes sociales, o para hacer actividades que sean de su interés, pero que no están relacionadas con la parte académica. Aunque algunos de ellos dedican una hora o dos para estudiar el fin de semana, uno de los entrevistados señaló: "Generalmente, me dedico a estudiar una o dos horas, uno que otro ejercicio de estadística, y, después, empiezo a vagar en internet".

De igual manera, los estudiantes que no asisten a este centro utilizan, también, excusas, tales como: "Pienso que es como una clase", "No coincide con mi horario, "No me interesa mejorar mi redacción", además de la ya 
mencionada "No tengo tiempo". La mayoría de ellos admitió tener problemas de redacción, pero no pretenden hacer algo para cambiarlo.

El siguiente aspecto trata de la percepción que tienen con respecto a sus faltas y aquella que tienen los tutores (Figuras 7 y 8 ).

A pesar de que los tutores son muy claros al mencionarles cuáles son sus respectivas falencias, en la encuesta podemos evidenciar que los aprendices ignoran estos comentarios.

Figura 7. Temas trabajados según estudiante.

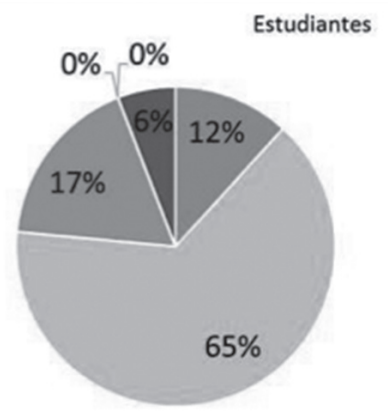

= Ortografia y gramática

" Redacción y articulación de aprrafos

= Formulación de tesis

- Planeación de la escritura

- Organización de ideas

- Estructura textual

Fuente. Elaboración propia.

Figura 8. Temas trabajados según tutores.

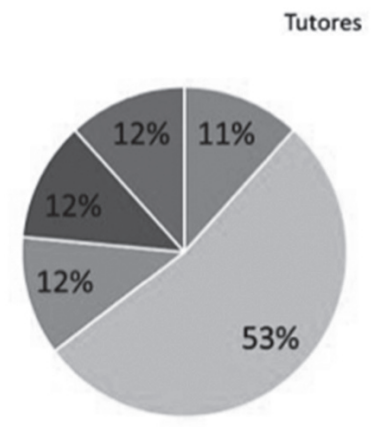

= Ortografia y gramática

" Redacción y articulación

de aprrafos

" Formulación de tesis

- Planeación de la escritura

- Organización de ideas

Fuente. Elaboración propia. 
Al contrastarlas, se comprueba que los estudiantes no creen tener problemas en no planear y organizar sus ideas antes de empezar a redactar un texto en particular: "Generalmente los alumnos traducen directamente su pensamiento al papel, no suelen planificar lo que van a escribir ni revisar lo que escriben" (Arias Gundín y García Sánchez, 2006; Fernández et al., 2010, citados por Aguilera y Boatto, 2013, p.140). Carlino explica la importancia de planear nuestros textos:

Es necesario que los docentes se ocupen de enseñar a planificar y a revisar lo escrito y ayuden a anticipar el punto de vista del destinatario, de modo que en este proceso no sólo se mejore el producto, sino que se guíe a sus autores a poner en práctica el escribir como herramienta para pensar los contenidos de cada materia. (2005, p. 31).

Así, comprobamos que los estudiantes no están anticipando el punto de vista que quieren plasmar en los escritos con claridad.

\section{Conclusiones}

espués de haber analizado los resultados que obtuvimos, podemos afirmar que nuestras suposiciones eran ciertas, ya que, como planteamos al principio, los estudiantes sí tienen otros motivos para asistir al Centro de Lectura y Escritura Académica muy diferentes de los de querer aprender. Recalcamos que la obtención de resultados fue un proceso lento, debido a que los asistentes por lo general lo hicieron una vez y, además, no se mostraban interesados en responder a nuestros cuestionamientos. Sin mencionar que el tiempo para la investigación fue ajustado y que este fue un factor determinante para no poder ampliar y tomar en cuenta otros elementos importantes, como la cultura, los profesores, el enfoque institucional, etc.

A partir de lo obtenido, concluimos que el hecho de asistir solo una vez y que ignoren los compromisos hechos con los tutores demuestra que a los estudiantes solo les interesa mejorar el trabajo con el que tienen dificultades, 
ya que es una nota importante y necesitan buenos resultados. Incluso al manifestar su satisfacción con la tutoría, al cumplir el objetivo que se habían propuesto, no ven necesario volver, incluso si tienen cosas que mejorar.

Ahora bien, los estudiantes hicieron un compromiso verbal con los tutores de seguir asistiendo a las tutorías de ser necesario, pero la única razón por la que aceptaron fue por cortesía. El negarse frente al ofrecimiento del tutor para continuar mejorando sus competencias lectoescritoras podría parecer descortés. Paradójicamente, un tutor expresó su descontento frente a un fenómeno que ha tenido que experimentar, y es que las personas no asistan a las citas previamente programadas y que tampoco las cancelen con anterioridad.

Frente a la excusa más recurrente que utilizan los estudiantes, que es la de no tener tiempo, se puede pensar que ellos no son conscientes de todo el que utilizan para su ocio y todo el que deberían utilizar para trabajar sobre cuestiones académicas. Esto denota incoherencias en los discursos de varios de los entrevistados, ya que, al preguntarles por qué no asistían, utilizaban la excusa mencionada, pero al preguntarles cómo utilizaban su tiempo libre, varios comentaron que en su mayoría lo utilizaban, bien para dormir, bien para gestionar sus redes sociales.

Asimismo, muchos de los que afirmaban tener una redacción aceptable, a su vez señalaban que no utilizaban ninguna estrategia para redactar. Sin embargo, como ya sé mencionó en los resultados, hacer un plan de escritura antes de plasmar las ideas en el papel no es solo importante sino necesario, y demuestra sus buenas habilidades para escribir. De la misma manera, los contenidos que leen los estudiantes, en buena parte, son los que se encuentran en redes sociales, y los textos que aparecen allí no tienen revisión formal, ni se tiene la certeza de que están bien redactados. Por ende, este tipo de lectura no influencia positivamente el proceso su escritura.

Al contrastar la percepción de tutores y alumnos, nos dimos cuenta de que los estudiantes no identifican en qué campos están fallando, porque simplemente no les interesa. El hecho de que asistan este centro para asegurar una buena nota pone en segundo plano el desarrollo de las habilidades de lectoescritura propias. Así, los estudiantes solo creen tener problemas de redacción en general, que es cierto, pero no buscan los que les competen 
directamente a ellos, es manifestar, en los que cada uno tiene dificultad; este es un proceso meramente personal.

Hay que manifestar que, entre los entrevistados, había opiniones divididas respecto de si un ingeniero, administrador, etc., necesita o no saber escribir correctamente. En cualquiera de los dos casos, no se demuestra interés por mejorar para ser más integral y competitivo, sino que, como explicamos, la nota impera en estas situaciones.

Nos damos cuenta de que hay un vacío en cuanto a aprendizaje autónomo y trabajo independiente. Aun estando en la modalidad de trabajo independiente, en la que el profesor da unas instrucciones y su rol es muy importante, no se cumple con las pautas mínimas que requiere, no se le dedica tiempo, y por esta razón los estudiantes están muy lejos de adentrarse en el mundo del aprendizaje autónomo, porque aún no tienen pensamiento crítico.

Finalmente, cabe manifestar que somos conscientes del poco recorrido de esta investigación y de las inconsistencias de la metodología utilizada, puesto que el grupo encuestado y entrevistado no fue muy grande y las dificultades que tuvimos, como el poco interés que expresaron los estudiantes en colaborarnos, limitaron los resultados. Sin embargo, creemos que es importante manifestar que lo que encontramos da una perspectiva inicial de lo que es el estudio autónomo y qué papel entra a desempeñar esto en este centro, y en general en las formas de estudio de los universitarios.

Por ello, estamos seguros de que esta investigación puede ampliarse, puede ir más allá y adentrarse en otros campos, como perspectivas de los profesores, una opinión mayor obtenida de encuestas en el ámbito de toda la universidad donde se puedan identificar cuáles son los hábitos de estudio, la organización del tiempo y la concepción de la escritura académica. Incluso comenzar a abrir puertas al análisis de la cultura del entorno social en el que estamos y si esta influye en diferentes aspectos en el ámbito universitario, como las formas de estudio, los hábitos de lectura, el interés por las calificaciones, etc. 


\section{Referencias}

Aguilera, M. S. y Boatto, Y.E. (2013). Seguir escribiendo... seguir aprendiendo. Zona Próxima, 18.

Arias Gundín, O. \& García Sánchez, J. (2006). El papel de la revisión en los modelos de escritura. Aula Abierta, 88, 37-52.

Ahumada, L. (2006). El discurso argumentativo de los estudiantes universitarios en los foros de discusión de un campus virtual: análisis del uso de marcadores discursivos. Psicoperspectivas, 5(1), 7-22. Recuperado de http://www.redalyc.org/html/1710/171016569001/

Alzate-Medina, G. M. y Peña-Borrero, L. B. (2010). La tutoría entre iguales: una modalidad para el desarrollo de la escritura en la educación superior. Universitas Psychologica, 9(1), 123-138.

Araya Venegas, L. (2007). ¿Qué nos pasa en escritura? Hipótesis sobre los problemas en la enseñanza de la lengua escrita. Lectura y Vida: Revista Latinoamericana de Lectura, 28(1), 6-14. Recuperado de https://goo.gl/ $\mathrm{n} 6 \mathrm{PdXj}$

Caouette, B. L. y Griggs, T. C. (2015). A compelling collaboration: The first year writing program, writing center, and directed self-placement. Praxis, 12(2), 17. Recuperado de http://www.praxisuwc.com/caouette-griggs-122/

Carlino, P. (2005). La escritura en el nivel superior. Escribir, leer y aprender en la universidad. Una introducción a la alfabetización académica. Buenos Aires: Fondo de Cultura Económica.

Carlino, P. (2008). Revisión entre pares en la formación de posgrado. Lectura y Vida: Revista Latinoamericana de Lectura, 29(2), 20-31.

Castells, M. (1997) La era de la información: economía, sociedad y cultura. (Vol. 1), Madrid: Alianza Editorial. 
Castro Azuara, M. C. y Sánchez Camargo, M. (2013). La expresión de opinión en textos académicos escritos por estudiantes universitarios. Revista Mexicana de Investigación Educativa, 18(57), 483-506. Recuperado de https://goo.gl/tQGCT3

Creswell, J. W. (2003). Research design: Qualitative, quantitative, and mixed methods approaches (2. ${ }^{\mathrm{a}}$ ed.). Londres: Sage Publications.

Cuervo Plazas, Z. L. (15 diciembre 2013). Necesitamos colegios públicos de buena calidad: rector de Icesi. En El País. Recuperado de http://www. elpais.com.co/cali/necesitamos-colegios-publicos-de-buena-dad-rector-deicesi.html

El Tiempo (2014). Los colombianos leen poco, prestado y regalado. Recuperado de http://www.eltiempo.com/multimedia/especiales/cuantoleen-los-colombianos/15606578/1/

Escorcia, D., Moreno, M., Campo, K. y Palacios, J. (2014). Enseñanza y evaluación de la escritura en la universidad. Zona Próxima, 20, 92-107. Recuperado de http://www.redalyc.org/html/853/85331022009/

Fernández G., Izuzquiza, M.V., Ballester, M.A., Marrón, M.P., Eizaguirre, M.D. \& Zanotti, F. (2010) Leer y escribir para aprender en los primeros años de la universidad. Lectura y Vida, 31(3), 62-71.

Flores Crespo, P. (2013). Investigaciones sobre el aprendizaje de los jóvenes. Revista Mexicana de Investigación Educativa, 18(57), 343- 347 Recuperado de https://goo.gl/QJBqQN

García Arroyo, M. y Quintana, H. (2008). El proceso de aprendizaje de la redacción en estudiantes universitarios: una mirada sobre sus propias reflexiones. Lectura y Vida: Revista Latinoamericana de Lectura, 29(3), 56-63. Recuperado de https://goo.gl/Bwc7fc 
Gómez Ruiz, M. Á., Rodríguez Gómez, G. y Ibarra Sáiz, M. S. (2013). COMPES: autoinforme sobre las competencias básicas relacionadas con la evaluación de los estudiantes universitarios. Estudios sobre Educación, 24. Recuperado de https://goo.gl/6Eumj3

Hossain, D. M. (2011). Qualitative research process. Postmodern Openings, 2(7), 143-156. Recuperado de http://postmodernopenings.com/wp-content/ uploads/2011/10/PO-7-102.pdf

Martín Zorraquino, M. A. y Portolés, J. (1999). Los marcadores del discurso. En I. Bosque, y V. Demonte (eds.), Gramática descriptiva de la lengua española (pp. 4051-4212). Madrid: Espasa Calpe.

Ministerio de Educación Nacional. Decreto 1295 2010. Por el cual se reglamenta el registro calificado de que trata la Ley 1188 de 2008 y la oferta y desarrollo de programas académicos de educación superior (20 abril 2010).

Molina, V. (2012). Writing across the curriculum in colombia: Current situation and challenges. Magis, 5(10).

Mondaca Becerra, L. A., Méndez Carrasco, A. P. y Rivadeneira Valenzuela, M. J. de L. (2015). "No es muletilla, es marcador, ¿cachái?”: Análisis de la función pragmática del marcador discursivo conversacional cachái en el español de Chile. Literatura y Lingüistica, 32, 233-258. https://dx.doi. org/10.4067/S0716-58112015000200013

Neuman, W.L. (1997) Social Research Methods Qualitative and Quantitative Approaches, (3rd edition). Boston: Allyn and Bacon.

Nussbaum, M. (2015). Discurso de Martha Nussbaum al recibir el doctorado honoris causa en UdeA. En Parque Explora. Recuperado de http://www. parqueexplora.org/visitenos/noticias/discurso-de-martha-nussbaum-alrecibir-el-doctorado-honoris-causa-en-udea/ 
Ochoa, L. (2014). El plagio y su relación con los procesos de escritura académica. Forma y Función, 27(2), 95-113. Recuperado de http://www. redalyc.org/articulo.oa?id=21935715003

Ordóñez, A. C. (8 julio 2017). Universitarios: desorientados y mal preparados. En Plaza Capital, 32. Recuperado de http://portal.urosario.edu.co/ pla_2004_2008/articulo.php?articulo $=1463$

Olaya Zúñiga, R. y Quimbayo Fandiño, L. R. (2014). Concepciones de aprendizaje autónomo de los estudiantes de Enfermería en dos instituciones de educación superior ubicadas en Bogotá (Tesis de maestría, Universidad Santo Tomás, Bogotá, Colombia). Recuperado de http://repository.usta. edu.co/handle/11634/415

Peláez Cárdenas, A. F. (2009). El aprendizaje autónomo y el crédito académico como respuesta a nuevo orden mundial en la educación universitaria. Cuadernos Latinoamericanos de Administración, 5(8), 51-66. Recuperado de http://revistas.unbosque.edu.co/index.php/cuaderlam/article/ view/1525/0

Román, E., Herrera, J. (2010). Aprendizaje centrado en el trabajo independiente. Educ.Educ., 13(1), 91-106. Disponible en: http://www.scielo.org.co/pdf/ eded/v13n1/v13n1a07.pdf

Rodríguez M., A., Solano, E., Martínez, A., Villar H. del, L. (2013). Lectura, escritura y calidad en la universidad colombiana. Zona Próxima, 18, 2-17. Recuperado de http://www.redalyc.org/articulo.oa?id=85328617002

Rojas-García, I. (2016). Aportes de la lingüística sistémico-funcional para la enseñanza de la lectura y la escritura en la educación superior. Educación y Educadores, 19(2), 185-204. Recuperado de http://www.redalyc.org/ $\mathrm{html} / 834 / 83446681001 /$

Vargas Franco, A. (2007). Escribir en la universidad: reflexiones y estrategias sobre el proceso de composición escrita de textos académicos. Cali, Colombia: Universidad del Valle. 
Vázquez, A. (2005) ¿Alfabetización en la universidad? Colección de Cuadernillos de actualización para pensar la Enseñanza Universitaria. 1(1). Universidad Nacional de Río Cuarto. Río Cuarto. Disponible en: http:// www.eemn 1 tsas.edu.ar/Autoevaluacion/Vasquez-Alfabetizacion $\% 20$ en\%201a\%20Universidad.pdf

Vega, M. (2015) La lectura digital de jóvenes sólo abarca textos breves $y$ efimeros. La Jornada. Recuperado de: http://www.jornada.unam. $\mathrm{mx} / 2015 / 11 / 05 /$ cultura/a03n1cul

Waller, S. C. (2002). A brief history of university writing centers: Variety and diversity. New Foundations. Recuperado de http://www.newfoundations. com/History/WritingCtr.html

Yesipov, V. P. (1981). El trabajo independiente de los alumnos en las clases. Moscú: Utstpedquis. 


\section{ANEXOS}

\section{Anexo 1. Encuesta}

Encuesta enviada por correo electrónico a los estudiantes que asistieron a este centro.

\section{Preguntas abiertas}

- ¿Por qué decidió acudir al Centro de Lectura y Escritura Académica?

- ¿Puede manifestar cuál es el propósito del Centro de Lectura y Escritura Académica?

- ¿Conoce usted los medios que utiliza el Centro de Lectura y Escritura Académica para ayudar a los estudiantes?

- Sí. ¿Cuáles?

$-\mathrm{No}$

- ¿Cuántas horas dedica a estudiar los temas de cada materia? Omita las horas de clase.

- ¿De qué manera se dio cuenta de que tenía dificultades a la hora de escribir o leer o ambas?

\section{Preguntas cerradas}

- ¿Alrededor de cuánto tiempo le dedicó a los escritos con los que tuvo dificultades y pidió ayuda?

- De 30 minutos a 1 hora

- De 1 a 2 horas

- De 2 horas a 3 horas

- 3 horas o más 
- ¿Cree que las competencias en lectura y escritura que recibió en el colegio fueron suficientes?

- Sí

$-\mathrm{No}$

- ¿Uno de sus pasatiempos es la lectura?

- Sí

- No

- ¿Cuáles de las siguientes opciones hacen parte del contenido que lee?

- Libros

- Revistas

- Artículos científicos

- Noticias

- Otro

- ¿Realiza escritos de práctica para entrenar su escritura?

- Sí

- No

- ¿Cree que es necesario que lleve un proceso y asistencia constante al Centro de Lectura y Escritura Académica?

- Sí

- No. ¿Por qué? 


\section{Anexo 2. Entrevistas}

Al ser de tipo semiestructurado, el formato es más libre, y a partir de las preguntas base, van surgiendo las demás de acuerdo con el desarrollo de la entrevista. A continuación, se incluyen las preguntas base de cada entrevista.

\section{Entrevista a los tutores del Centro de Lectura y Escritura Académica}

- ¿Ha considerado dar consejos de estudio?

- ¿Por qué considera ella que los estudiantes no saben escribir?

- ¿Qué lo hizo ser tutor? ¿Qué características que los otros no tienen te llevaron a convertirte en tutora?

- ¿Logra identificar cuando alguien no está muy interesado en aprender?

- ¿Ves mejora en aquellos que han repetido sesiones?

- ¿Ha considerado dejar ejercicios de repaso a los asistentes?

\section{Entrevista a los no asistentes}

- ¿Conoces el Centro de Lectura y Escritura Académica?

- ¿Qué te imaginas que puedes encontrar allá?

- ¿Cuántos créditos matriculaste este semestre?

- ¿Cuál es la materia más difícil este semestre?

- ¿Estudias para esa materia?

- ¿Qué métodos de estudio utilizas?

- ¿Haces ejercicios de escritura?

- ¿Cuántos libros lees al año, en promedio?

- ¿Escribes de manera autónoma?

- ¿Qué haces en tu tiempo libre?

- ¿Qué haces en las tardes cuando llegas de estudiar? 


\section{Entrevista a los asistentes}

- ¿Por qué motivo asististe al Centro de Lectura y Escritura Académica?

- ¿Cuántas veces has ido?

- ¿Por cuál materia has pedido ayuda?

- ¿Volverías a pedir tutoría?

- ¿Cuántos créditos matriculaste este semestre?

- ¿Cuál es la materia más difícil este semestre?

- ¿Estudias para esa materia?

- ¿Qué métodos de estudio utilizas?

- ¿Haces ejercicios de escritura?

- ¿Cuántos libros lees al año, en promedio?

- ¿Escribes de manera autónoma?

- ¿Qué haces en su tiempo libre?

- ¿Qué hace en las tardes cuando llega de estudiar? 
